\title{
Effectiveness of Environmental Control for the Ultrastructural Study of Buttery Materials using an Air Protection Holder and NB5000/HD-2700 System.
}

\author{
T. Sato*, M. Kudo*, H. Okushima*, A. Morikawa*, Y. Kuroda*, T. Onishi** and T. Agemura** \\ * Hitachi High-Technologies Corp., 11-1, Ishikawa, Hitachinaka-shi, Ibaraki, 312-0057, Japan \\ ** Hitachi High-Technologies Corp., 882, Ichige, Hitachinaka-shi, Ibaraki, 312-8504, Japan
}

Focused ion beam (FIB) technique has been used for the specimen preparation of many kinds of materials, such as semiconductor, ceramics, metal and polymer by a scanning transmission electron microscope (STEM). Recently, this technique has been applied to the new functional materials with huge potential such as organic display and battery material. Especially, Lithium ion battery (LIB) has become widely used for the power supply of personal computer and mobile phone [1]. But these functional materials have the possibility to change the structure by the amount of fluid and oxygen of the atmosphere, so it is difficult to analyze their intact structures by traditional specimen preparation method. Therefore, in this study, environmental control devices, such as a glove box and an air protection holder has been developed to protect air exposure during the specimen preparation, and the technology was applied for the LIB. Here, the effectiveness of environmental control for preparation of LIB and its observation by STEM has been shown. The specimen preparation procedure for STEM observation has been done as follows. At first, nitrogen gas was induced to the globe box trough the tube for approximately $10 \mathrm{~min}$ and, nitrogen concentration in the globe box achieved $100 \%$. Specimen was mounted on the air protection holder for eucentric stage inside the globe box. And then, this holder was inserted in FIB system kept on air protecting. In this system, the globe box was connected to the NB5000 FIB-SEM, so the specimen was completely intercepted from atmosphere and prepared by the microsampling method (FIG.1) [2]. Specimen was thinned by accelerated $40 \mathrm{kV}$ Ga ion. The thin specimen was inserted in HD-2700 STEM instrument. This holder is compatible to FIB system and STEM instrument so, it is no necessary to re-mount. Specimen was studied using the Hitachi HD-2700 dedicated STEM equipped with the Field Emission Gun with the accelerating voltage $200 \mathrm{kV}$ (FIG.2) [3]. FIG.3 shows the surface SIM images of negative electrode of LIB taken at air protection (a) and atmospheric pressure (b). Many particles about 10-20 $\mu \mathrm{m}$ size were clearly observed under the air protection condition, but the surface structure was metamorphose atmospheric pressure. FIG.4 shows the cross section STEM images of the thinned LIB specimen taken at air protection (a) and atmospheric pressure (b). Fine structures were clearly observed under the air protection condition, but their structures were not clearly observed atmospheric pressure. This result showed that the air-protection holder was useful to intercept air and fabricate thin specimen of lithium ion battery. The results also emphasized that this technology provides effective capability to study of new functional materials sensitive to the amount of fluid and oxygen.

References

[1] Y. Nagao, H. Sakaguchi, and T. Esaka, J. Electronchem. Soc., 151, P.1572 (2004)

[2] T. Ohnishi, and T. Ishitani, JP Patent 2774884 and US patent 5270552

[3] H. Inada, L. Wu, J. Wall, D. Su, \& Y. Zhu, J. of Electron Microsc. 58, 111-122 (2009) 


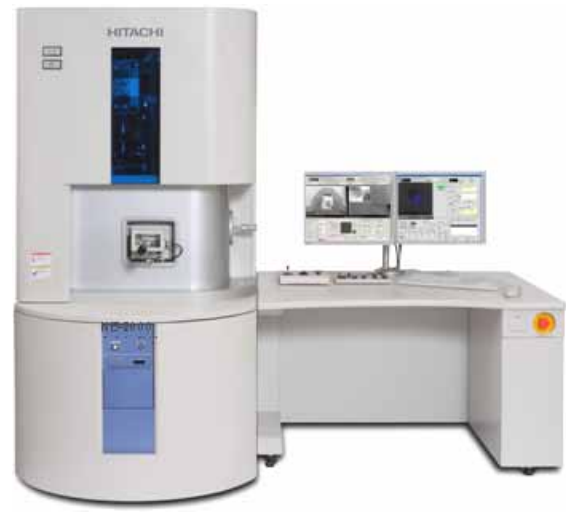

FIG.1 A general view of NB5000 FIB-SEM system.

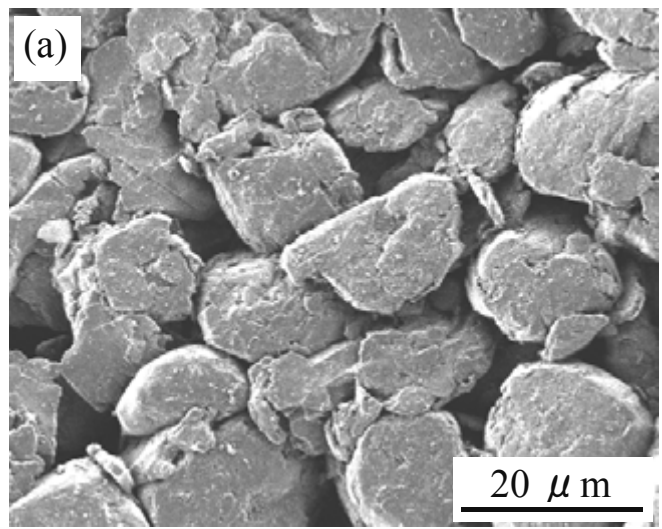

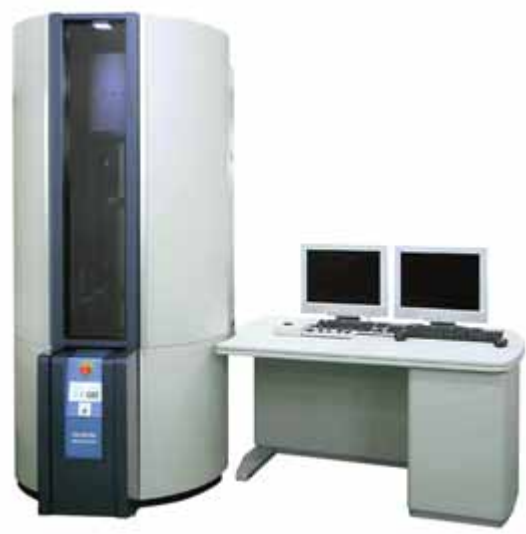

FIG.2 A general view of HD-2700 STEM.

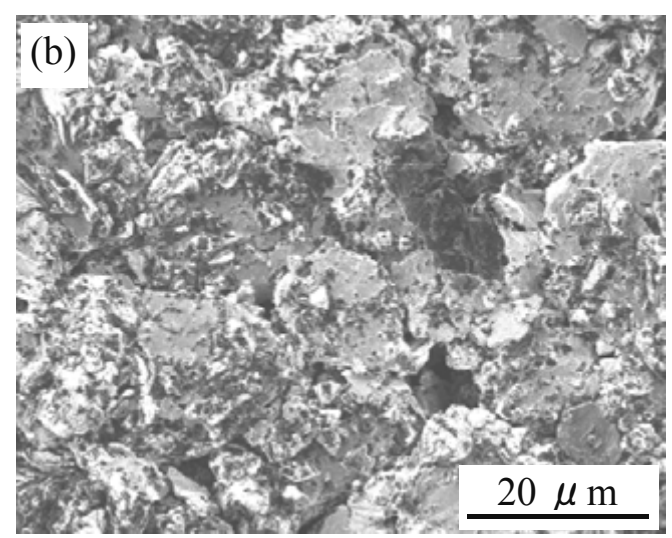

FIG.3 Surface SIM images of negative electrode of LIB at air protection (a) and atmospheric pressure (b).
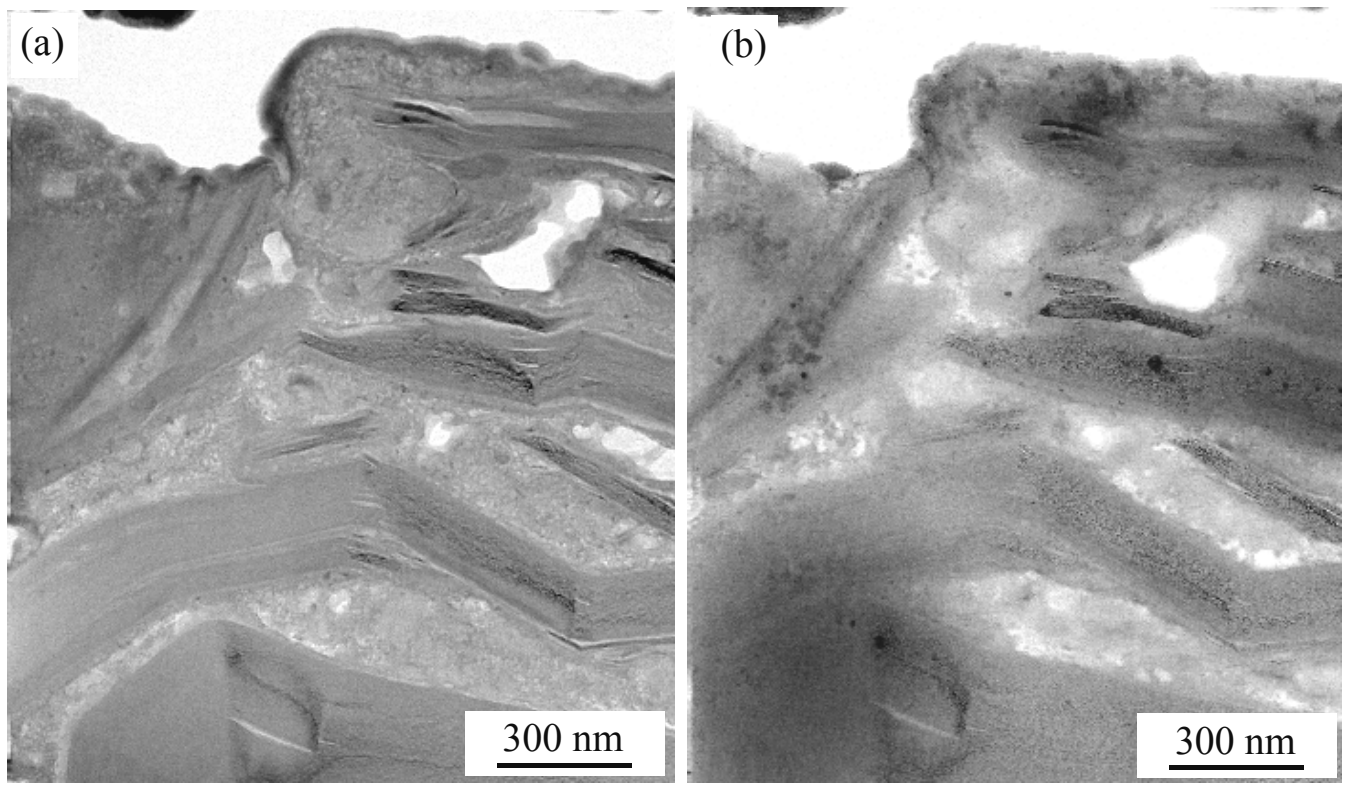

FIG.4 Cross section STEM images of thinned LIB at air protection (a) and atmospheric pressure (b). 